\title{
A Mathematical Biologist's Guide to Absolute and Convective Instability
}

\author{
Jonathan A. Sherratt • Ayawoa S. Dagbovie • \\ Frank M. Hilker
}

Received: 29 June 2013 / Accepted: 8 October 2013 / Published online: 22 November 2013

(C) Society for Mathematical Biology 2013

\begin{abstract}
Mathematical models have been highly successful at reproducing the complex spatiotemporal phenomena seen in many biological systems. However, the ability to numerically simulate such phenomena currently far outstrips detailed mathematical understanding. This paper reviews the theory of absolute and convective instability, which has the potential to redress this inbalance in some cases. In spatiotemporal systems, unstable steady states subdivide into two categories. Those that are absolutely unstable are not relevant in applications except as generators of spatial or spatiotemporal patterns, but convectively unstable steady states can occur as persistent features of solutions. The authors explain the concepts of absolute and convective instability, and also the related concepts of remnant and transient instability. They give examples of their use in explaining qualitative transitions in solution behaviour. They then describe how to distinguish different types of instability, focussing on the relatively new approach of the absolute spectrum. They also discuss the use of the theory for making quantitative predictions on how spatiotemporal solutions change with model parameters. The discussion is illustrated throughout by numerical simulations of a model for river-based predator-prey systems.
\end{abstract}

Keywords Absolute stability · Partial differential equations · Pattern formation · Spatiotemporal patterns $\cdot$ Review $\cdot$ Survey

\footnotetext{
J.A. Sherratt $(\varangle) \cdot$ A.S. Dagbovie

Department of Mathematics and Maxwell Institute for Mathematical Sciences, Heriot-Watt University, Edinburgh EH14 4AS, UK

e-mail: j.a.sherratt@hw.ac.uk
}

\section{A.S. Dagbovie \\ e-mail: ad195@hw.ac.uk}

F.M. Hilker

Centre for Mathematical Biology and Department of Mathematical Sciences, University of Bath, Bath BA2 7AY, UK

e-mail: f.hilker@bath.ac.uk 


\section{Introduction}

Almost all undergraduate courses in mathematical biology include a section on ordinary differential equation (ODE) models. The central player in the course material is the stability of steady states. Students learn that only (locally) stable steady states are biologically significant. Later in their careers, students are introduced to partial differential equation (PDE) models. Again, the stability of (homogeneous) steady states plays a central role. In particular, the first exposure that many students receive to biological pattern formation is the Turing mechanism, in which a homogeneous steady state that is stable in the kinetic ODEs is destabilised by the addition of diffusion terms. However, steady states that are unstable in the kinetic ODEs are almost never mentioned. As a result, there is a widespread assumption that unstable steady states are not biologically significant as PDE solutions. This is not true. In fact, such steady states fall into one of two categories. If they are "absolutely unstable", then they are indeed not biologically significant, except perhaps as providing the mathematical origin of spatial or spatiotemporal patterns. But "convectively unstable" steady states can be an important and persistent feature of PDE solutions. The objective of this paper is to explain the concepts of absolute and convective instability and the related concepts of remnant and transient instability, to illustrate their implications for PDE models of biological systems, and to summarise methods for distinguishing different types of instability in practice.

We begin with an illustrative example: the invasion of a prey population by predators. This problem has been addressed in many modelling studies. Simple invasions correspond to a standard transition front (e.g. Owen and Lewis 2001), but there is now a considerable body of work on invasions that leave more complex spatiotemporal phenomena in their wake: see, for example, Sherratt et al. (1995), Petrovskii and Malchow (2000), Morozov et al. (2006), Merchant and Nagata (2010, 2011). We will focus on the spatially extended Rosenzweig-MacArthur (1963) model for predatorprey interaction, and for clarity all of the numerical simulations in this paper will use this model. We give the equations in a dimensionless form:

$$
\begin{aligned}
\text { predators } \frac{\partial p}{\partial t} & =\overbrace{d \frac{\partial^{2} p}{\partial x^{2}}}^{\text {dispersal }}+\overbrace{c \frac{\partial p}{\partial x}}^{\text {advection }}+\overbrace{(\mu / b) h p /(1+\mu h)}^{\begin{array}{c}
\text { benefit from } \\
\text { predation }
\end{array}}-\overbrace{p / a b}^{\text {death }}, \\
\text { prey } \frac{\partial h}{\partial t} & =\underbrace{\frac{\partial^{2} h}{\partial x^{2}}}_{\text {dispersal }}+\underbrace{c \frac{\partial h}{\partial x}}_{\text {advection }}+\underbrace{h(1-h)}_{\begin{array}{c}
\text { intrinsic } \\
\text { birth and death }
\end{array}}-\underbrace{\frac{\mu p h}{1+\mu h}}_{\text {predation }} .
\end{aligned}
$$

Here, $p$ and $h$ denote predator and prey densities, which depend on space $x$ and time $t$. Here, and throughout this paper, we restrict attention to one space dimension: The theory of absolute stability is not yet fully developed in higher dimensions. Most predator-prey studies do not include advection terms, but allowing $c \neq 0$ enables a clearer illustration of some of the concepts we will be discussing. Advection of this type arises naturally in river-based predator-prey systems (Hilker and Lewis 2010). The prey consumption rate per predator is an increasing saturating function of the 

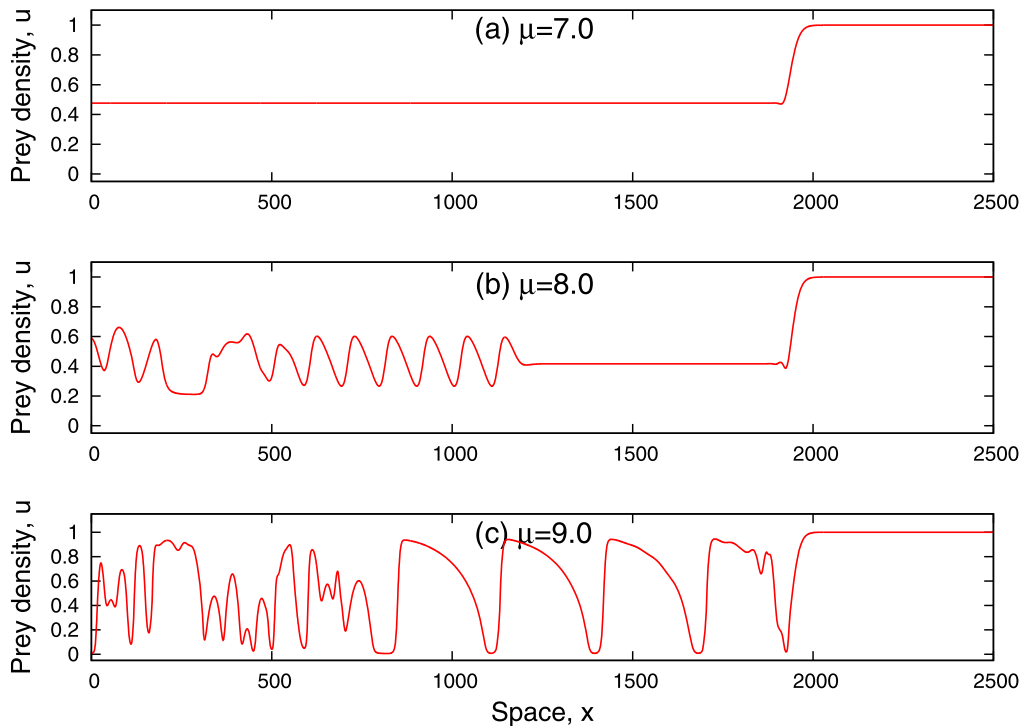

Fig. 1 Numerical simulation of predators invading prey using the Rosenzweig-MacArthur (1963) model (1a), (1b) without advection. At time $t=0$, the system was in the prey-only steady state $(1,0)$ except for a small predator density near the left-hand boundary. The solution is plotted at $t=4000$. In (a), the parameters are such that the coexistence steady state is stable, and the invasion consists of a simple transition front, connecting the prey-only state and the coexistence state. In (b), the coexistence state persists in a plateau behind the leading front, with spatiotemporal oscillations further back. In (c) there is a periodic travelling wave immediately behind and moving at the same speed as the leading front, with more irregular oscillations further back. Note that for Eq. (1a), (1b), the existence of both steady state to steady state and steady state to wavetrain transition fronts has been proved by Dunbar (1986) in the limit as $d \rightarrow \infty$, with extensions to $d$ finite and sufficiently large by Fraile and Sabina (1989) in the latter case. The parameter values were $a=1.3, b=4, c=0, d=2$ and (a) $\mu=7$; (b) $\mu=8$; (c) $\mu=9$. The equations were solved numerically using a semi-implicit finite difference method with a grid spacing of 0.5 and a time step of 0.01 . We solved on the domain $0<x<2500$, with zero flux conditions $p_{x}=h_{x}=0$ at both boundaries

prey density with Holling type II form: $\mu>0$ reflects how quickly the consumption rate saturates as prey density increases. Parameters $a>0$ and $b>0$ are dimensionless combinations of the birth and death rates; Details of the nondimensionalisation are given in Appendix A of Smith et al. (2008), and in many textbooks. The parameter $d>0$ is the ratio of predator to prey dispersal coefficients. The value of $d$ will be significantly greater than one for most mammalian systems (e.g. Brandt and Lambin 2007) and also for macroscopic marine species (Wieters et al. 2008). However, $d$ will typically be closer to one for aquatic microorganisms (Hauzy et al. 2007). Provided $a>1+1 / \mu$, Eqs. (1a), (1b) have a unique homogeneous coexistence steady state $\left(h_{s}, p_{s}\right)$ where $h_{s}=1 /(a \mu-\mu)$ and $p_{s}=\left(1-h_{s}\right)\left(1+\mu h_{s}\right) / \mu$, which is stable as a solution of the kinetic ODEs only if $\mu<\mu_{\text {crit }}=(a+1) /(a-1)$.

Figure 1 illustrates simulations of the invasion of a prey population by predators using (1a), (1b) with no advection, i.e. $c=0$. For $\mu<\mu_{\text {crit }}$, invasion takes the form of a simple transition wave, with the prey-only state $(1,0)$ ahead of the wave and the stable coexistence state $\left(h_{s}, p_{s}\right)$ behind it (see Fig. 1a). But as $\mu$ is increased above $\mu_{\text {crit }}$, so that the coexistence steady state becomes unstable, this steady state 
does not suddenly disappear (see Fig. 1b, c). Rather, for a range of $\mu$ values above $\mu_{\text {crit }}$ there is plateau behind the front in which the solution is at the coexistence state, with spatiotemporal oscillations further back. This plateau is a persistent feature of the invasion profile, despite the fact that the coexistence steady state is unstable. Malchow and Petrovskii (2002) termed this phenomenon "dynamical stabilization", and the key to understanding it is the concept of absolute vs. convective instability. In Sect. 2, we explain these terms, and in Sect. 3 we give an example of their use in explaining qualitative transitions in solution behaviour. In Sect. 4, we summarise the historical development of the theory, and in Sect. 5 we describe how to distinguish different types of instability using the relatively new approach of the "absolute spectrum". In Sect. 6, we explain how the theory can be used to make quantitative predictions on how aspects of spatiotemporal behaviour change with model parameters. In Sect. 7, we summarise our discussion, consider its application to a wider class of solutions and place the concept of convective and absolute instabilities into the context of ecology, especially with respect to perturbations in stream ecosystems and biological pattern formation.

\section{Absolute and Convective Stability}

In a temporal system, stability is a relatively simple concept: A solution is (locally) stable if any small perturbation decays. The same is true in a spatiotemporal system: An unstable steady state is defined as one for which some small perturbations grow over time. However, the situation is complicated by the fact that spatially localised perturbations may move while they are growing. Consequently, it is possible that a perturbation may decay at the location at which it is applied, even though it is growing overall (Fig. 2a, b). "Convective instability" occurs when all growing perturbations (linear modes) move while they are

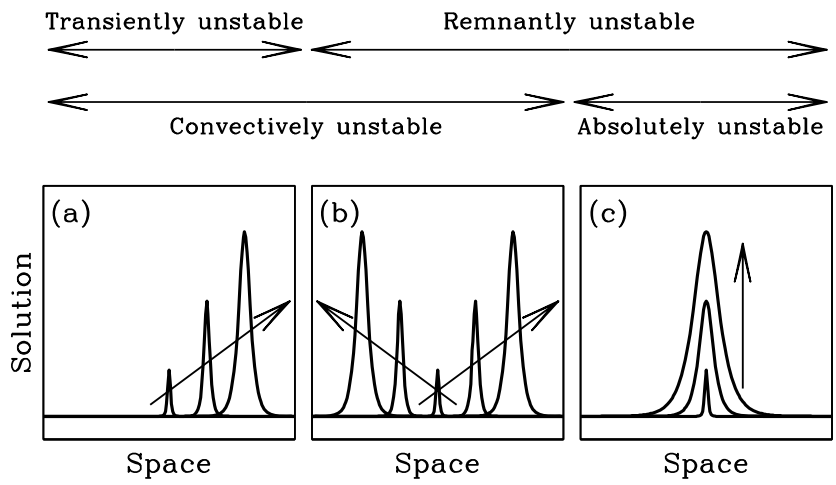

Fig. 2 A schematic illustration of different types of instability. (a) In transient convective instability, all unstable linear modes move in a single direction while they are growing. (b) In non-transient convective instability, all unstable linear modes move while they are growing, but in both directions. (c) In absolute instability, there is a stationary unstable linear mode, so that there are perturbations that grow at the location at which they are applied. The figure is adapted from Fig. 1 of Sandstede and Scheel (2000b) 

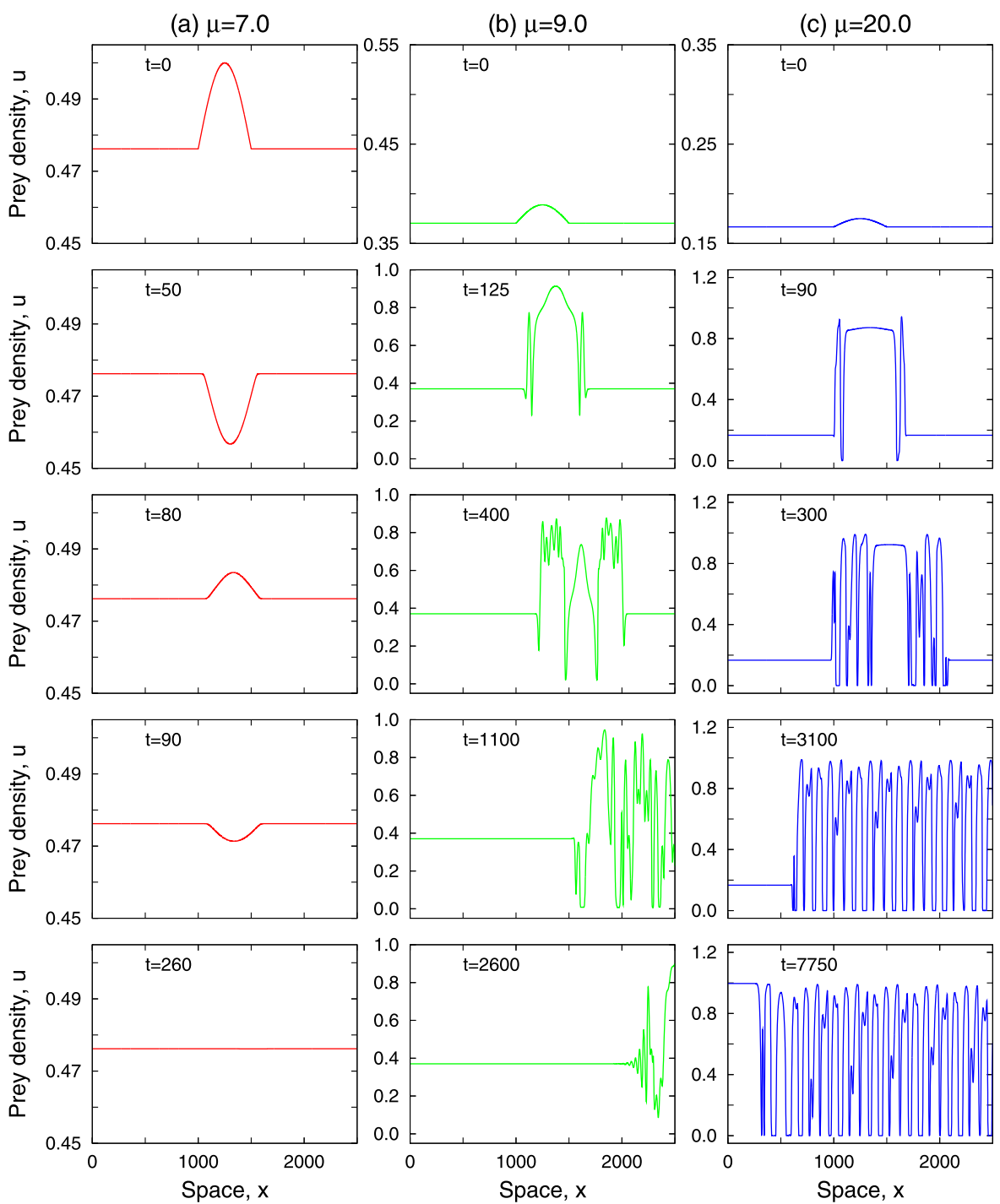

Fig. 3 Numerical simulations of the Rosenzweig-MacArthur model (1a), (1b) following a small perturbation to the co-existence steady state. (a) For $\mu=7$, the steady state is stable and the perturbation decays. (b), (c) For $\mu=9$ and $\mu=20$, the steady state is unstable. In the former case, the perturbation grows but simultaneously moves across the domain, while in the latter case it grows at the point at which it was initially applied. The other parameters were $a=1.3, b=4, c=-1, d=2$. The initial condition in each case was $(h, p)=\left(h_{s}, p_{s}\right) \cdot[1+0.05 \sin (\pi x / 1000)]$ if $1000<x<1500$ and $(h, p)=\left(h_{s}, p_{s}\right)$ otherwise. The equations were solved numerically on $0<x<2500$ as in Fig. 1, with the same space and time discretisations, with Danckwert's (zero Neumann) boundary conditions at both ends

growing. In contrast, "absolute instability" denotes the situation in which there are perturbations (linear modes) that grow at the location at which they are applied (Fig. 2c). 
In Fig. 3, we illustrate these two types of instability using simulations of (1a), (1b). We apply a small perturbation, localised around the centre of the domain, to the coexistence steady state $\left(h_{s}, p_{s}\right)$. For $\mu<\mu_{\text {crit }}=7.67$, the steady state is stable and the perturbation decays. For $\mu=9$, the perturbation grows but moves in the positive $x$ direction, decaying at its original location, while for $\mu=20$ the perturbation grows at its original location. This illustrates the fact that for the values of $a, b, c$, and $d$ used in the figure, the coexistence steady state is convectively unstable for $\mu=9$ and absolutely unstable for $\mu=20$. Note that our choice of $\mu$ as a control parameter is essentially arbitrary, and similar changes can be induced by changes in $a, b, c$, or $d$, for appropriate values of the other parameters.

Practical applications of PDE models in mathematical biology almost always occur on finite domains, and then boundary conditions must be considered when drawing conclusions about stability. If one considers (1a), (1b) on a domain with periodic boundary conditions, and with a value of $\mu$ giving convective instability, a moving growing perturbation will eventually reach the right-hand boundary. It will then reenter at the left-hand boundary and continue growing (and moving) (Fig. 4a). Therefore, the steady state is unstable in this case. In fact, it is a general result that a steady state that is unstable as a solution of the kinetic ODEs is also unstable as a PDE solution on a finite domain with periodic boundary conditions (Sandstede and Scheel 2000b). However, for the same parameter values as used in Fig. 4a but with separated boundary conditions such as Neumann, Dirichlet, or Robin, the coexistence steady state of (1a), (1b) is stable. This is because the growing perturbation moves in the positive $x$ direction until it reaches the right-hand boundary, where it is absorbed (Fig. 4b). Note that the simulations in Fig. 4 are intended as mathematical illustrations, rather than as simulations of a realistic scenario for a river-based predator-prey system. In the context of that application, a Dirichlet boundary condition corresponds to a hostile boundary such as a waterfall or a region containing toxic waste water, while a zero-flux condition is of Robin type. A zero Neumann boundary condition is known as a Danckwert boundary condition and corresponds to a long river in which the downstream boundary has little influence (Lutscher et al. 2006; Nauman 2008 \$9.3.1; Hilker and Lewis 2010).

For most equation systems, a convectively unstable steady state is stable on a finite domain with separated boundary conditions, as is the case for (1a), (1b). However, this will not be the case if there are growing perturbations that travel to the left and right simultaneously, while decaying at their original location: this is known as "non-transient convective instability" (Sandstede and Scheel 2000b; Fig. 2). Then on a finite domain the growing perturbations will typically be reflected by the boundaries rather than being absorbed, so that the steady state is unstable. The distinction between transient and non-transient convective instabilities was first recognised by Proctor and co-workers (Worledge et al. 1997; Tobias et al. 1998; Fox and Proctor 1998), and Sandstede and Scheel (2000b) argue that it is more instructive to distinguish between transient and remnant instabilities than between convective and absolute instabilities. Here, the term "remnant instability" means an instability that is either absolute or non-transient convective. One therefore has the following result:

on a large finite domain with separated boundary conditions, transiently unsta-

ble steady states are stable, while remnantly unstable steady states are unstable 
Fig. 4 Numerical simulations of the Rosenzweig-MacArthur model (1a), (1b) following a small perturbation to the coexistence steady state for $a=1.3, b=4, c=-1, d=2$, $\mu=9$ with (a) periodic boundary conditions; (b) Dirichlet conditions $(h, p)=\left(h_{s}, p_{s}\right)$ at both boundaries. In both cases, the perturbation grows but travels in the positive $x$ direction. In (a), when the growing perturbation reaches the right hand boundary it re-enters the domain at the left-hand boundary and continues to move, reaching the original site of perturbation with a greater amplitude than it had initially. The repetition of this process results in the steady state being unstable. However, in (b), when the growing perturbation reaches the right-hand boundary it is absorbed, so that the steady state is stable. The initial conditions and the values of the other parameters were as in Fig. 3, and the numerical method was as in Fig. 1, with the same space and time discretisations (a) Periodic BCs

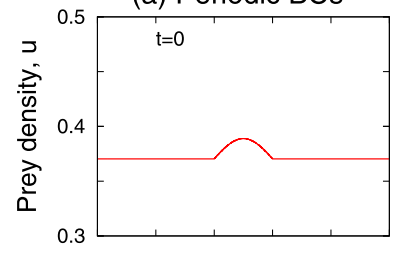

(b) Dirichlet BCs
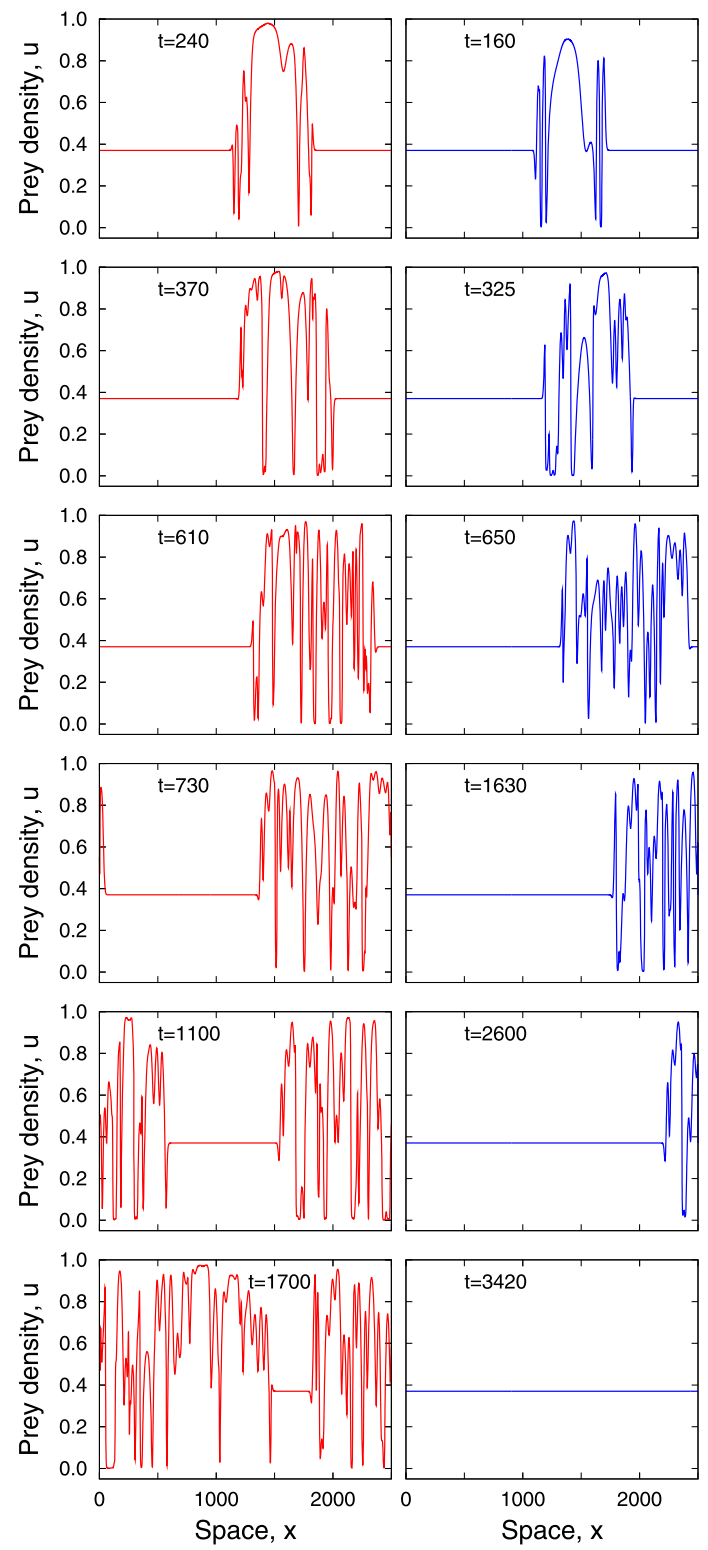
(see Sandstede and Scheel 2000 b for a more precise statement). Examples of nontransient convective instabilities are given in Sandstede and Scheel (2000b, Example 2 in Sect. 3.3) and (Rademacher et al. 2007, Sect. 5.2). However, we are not aware of an example from a biological application. This means that the distinctions between absolute and convective instabilities is the same as that between remnant and transient instabilities in all mathematical biology models in which these issues have been investigated at the time of writing.

\section{An Illustrative Example}

We now present an illustration of different qualitative solution forms resulting from a steady state being convectively or absolutely unstable. We consider (1a), (1b) on a finite domain with the zero flux condition $h_{x}+c h=d p_{x}+c p=0$ at the left-hand boundary and the Danckwert condition $h_{x}=p_{x}=0$ at the right-hand boundary. An example situation in which such boundary conditions would be relevant is a long section of river in which the left hand boundary corresponds to the river's source (Lutscher et al. 2006). Initially, we set the populations to their coexistence steady state levels in the interior of the domain. This steady state is incompatible with the zero flux boundary condition, and the predators are gradually washed out of system (Fig. 5; Hilker and Lewis 2010). This occurs via a transition front moving across the domain, so that the prey-only steady state appears to "invade" the co-existence steady state. In Fig. 5a, the co-existence steady state is stable, and the transition front is of a simple type. However, in Fig. 5b, the steady state is (transiently) convectively unstable. The tail of the transition front applies perturbations to the coexistence steady state ahead of it, and these perturbations all travel in the positive $x$ direction as they grow. Therefore, the solution remains at (or very close to) the steady state immediately ahead of the front, with spatiotemporal oscillations developing further to the right, where the perturbations have grown sufficiently large to have a significant effect. In Fig. 5c, the steady state is absolutely unstable. Then there are both stationary and moving linear modes in the perturbation applied to the co-existence steady state by the transition front. Therefore, spatiotemporal oscillations develop everywhere ahead of the front (Fig. 5c).

This simple example illustrates the way in which convective and absolute instability lead to qualitatively different solutions. Within the convectively unstable parameter regime the width of the region in which the populations are at the co-existence steady state is a decreasing function of $\mu$. Intuitively, as $\mu$ increases the perturbations imposed on the steady state by the invasion front travel away from the front more slowly, and grow more quickly. In Sect. 6, we will show how a detailed study of the movement and growth of small perturbations can be used to calculate the dependence on $\mu$ of the width of the steady state region.

\section{A Brief History of Absolute Stability}

The concept of absolute stability was initially developed in the context of plasma physics, with the first detailed presentation being the monograph by Briggs (1964). 
(a) $\mu=7.0$
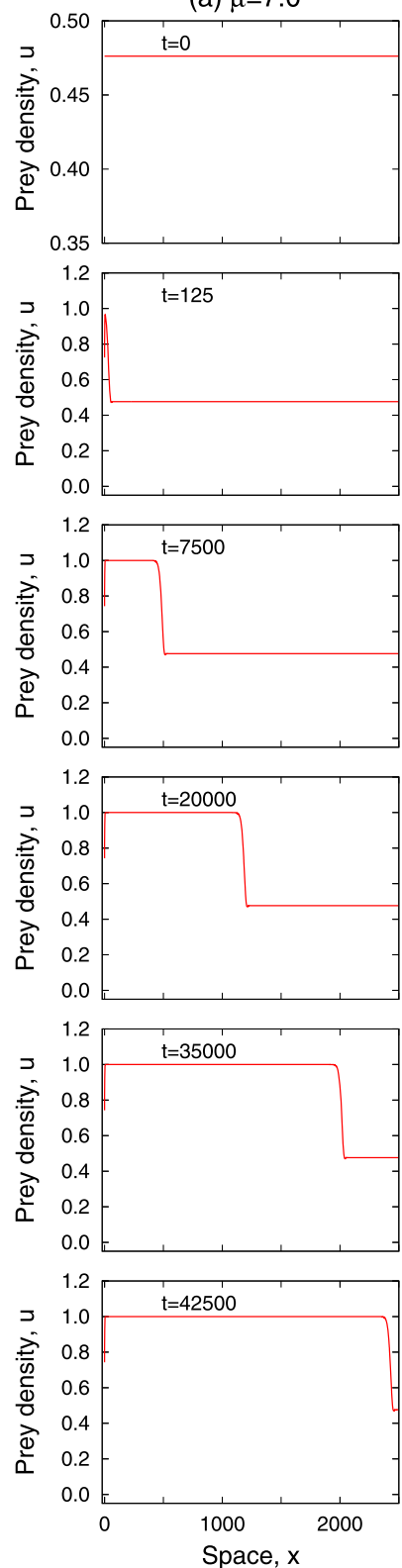

(b) $\mu=8.0$
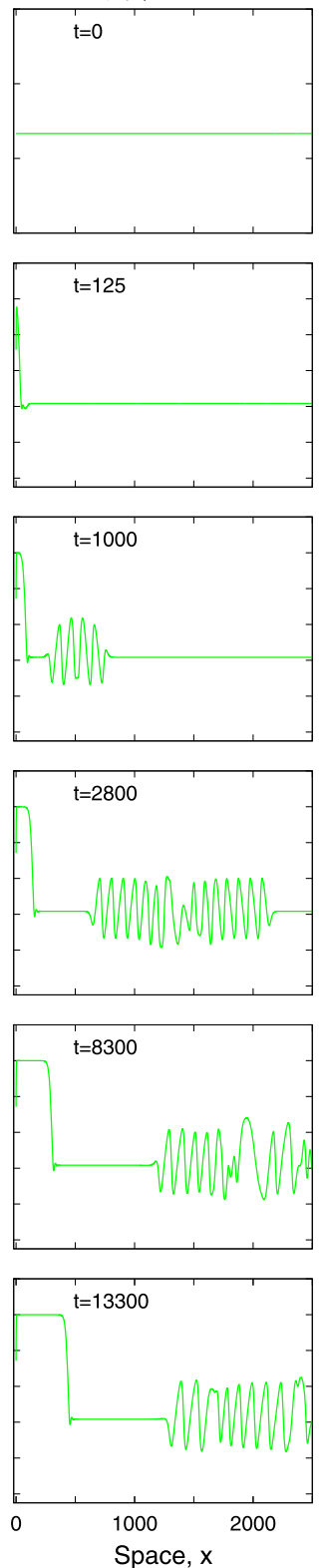

(c) $\mu=8.6$
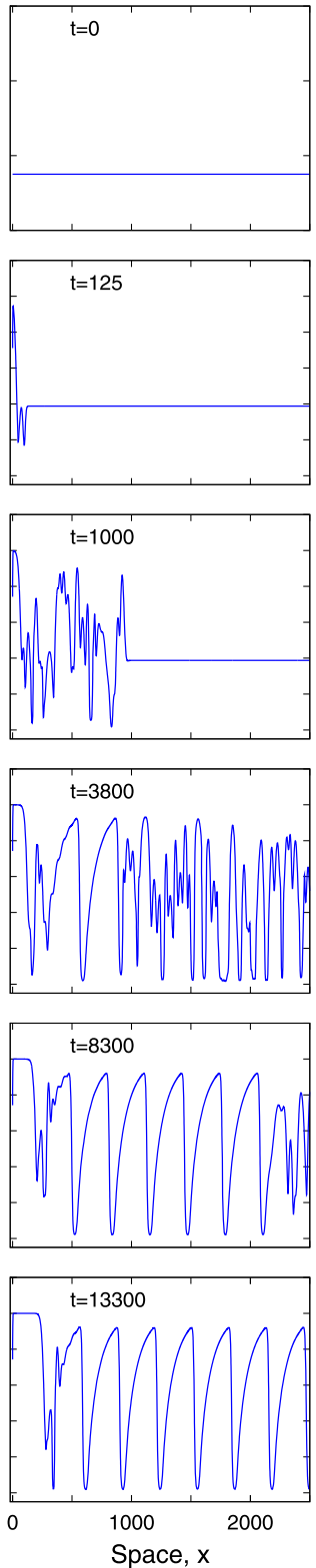

Fig. 5 Numerical simulations of the Rosenzweig-MacArthur (1963) model (1a), (1b) with the zero flux condition $h_{x}+c h=d p_{x}+c p=0$ at the left-hand boundary and the Danckwert condition $h_{x}=p_{x}=0$ at the right-hand boundary. Initially, the solution is at the co-existence steady state, but this is incompatible with the left-hand boundary condition, and a transition front develops that replaces the co-existence steady state with the prey-only state. The co-existence steady state is stable in (a) and unstable in (b), (c). Therefore, the transition front is of a simple type in (a) while in (b), (c) spatiotemporal oscillations develop, either some distance downstream (b) or immediately ahead of the front (c). The parameter values were $a=1.3, b=4, c=-0.5, d=2$ and (a) $\mu=7$, (b) $\mu=8$, (c) $\mu=8.6$. The numerical method was as in Fig. 1, with the same space and time discretisations 
In the subsequent decades the ideas have come into common usage in fluid dynamics (reviewed by Huerre and Monkewitz 1990 and Chomaz 2005) and spiral wave breakup (e.g. Aranson et al. 1992; Sandstede and Scheel 2000a; Wheeler and Barkley 2006). However, they remain almost entirely absent from the literature on applications of mathematics to chemistry, ecology, biology, and medicine.

A fundamental issue is how one calculates whether an unstable steady state is convectively or absolutely unstable. The original approach of Briggs (1964) was to solve the linear equations governing small perturbations by Fourier and Laplace transforms, and to consider the large time asymptotics of the inverse transforms. Eigenvalues satisfying a criterion known as the "pinching condition" play a special role in this asymptotic behaviour, because they prevent appropriate deformation of the Fourier integration contour. Briggs (1964) showed that the condition for convective instability was that all eigenvalues satisfying the pinching condition have negative real part. Briggs' (1964) work assumed that the integrand in the inverse Fourier transform has only first order poles; his results were extended to include higher order poles by Brevdo (1988), and to spatially and temporally periodic solutions by Brevdo and Bridges (1996, 1997a).

Numerical implementation of the Briggs-Brevdo-Bridges criterion is relatively difficult. Brevdo and co-workers (Brevdo 1995; Brevdo et al. 1999) developed a procedure based on numerical continuation of the location of a saddle point of a particular function of the eigenvalues; this saddle point gives the leading order contribution to the long-time asymptotics of the inverse Fourier transform. More recently, Suslov (2001, 2006, 2009), Suslov and Paolucci (2004) extended this approach to give an automatic search algorithm for calculating the convective-absolute stability boundary in parameter space. Brevdo's method is complicated, and Suslov's (highly ingenious) method is extremely complicated; neither is really suitable for non-specialists.

Fortunately, a new approach to the determination of convective/absolute instability has been developed by Sandstede and Scheel (2000b). Building on the work of Beyn and Lorenz (1999) on exponential dichotomies, Sandstede and Scheel (2000b) introduced the notion of the "absolute spectrum". This term is a slight misnomer, since the absolute spectrum is not the set of eigenvalues of any linear operator. However it serves a similar purpose in practice: It is a set of eigenvalues, and different types of instability can be distinguished by whether the absolute spectrum does/does not cross into the right-hand half of the complex plane. In practice, the absolute spectrum provides a relatively straightforward means of calculating absolute stability, even for the non-specialist.

\section{The Absolute Spectrum}

The first step in considering stability of a homogeneous steady state is to linearise the governing PDEs about the steady state. In the standard way, one then looks for solutions of these linear equations that are proportional to $e^{\lambda t+i k x}$; for non-trivial solutions, this leads to a dispersion relation $\mathcal{D}(\lambda, k)=0$ to be satisfied by $\lambda$ and $k$. We denote by $N$ the order of $\mathcal{D}$ as a polynomial in $k$; thus for (1a), (1b), $N=4$. To calculate stability of the steady state, one considers values of $\lambda$ satisfying $\mathcal{D}(\lambda, k)=0$ 
with $k \in \mathbb{R}$; the steady state is stable if and only if all such $\lambda$ 's (except possibly $\lambda=0$ ) have $\operatorname{Re} \lambda<0$.

The set of values of $\lambda$ for which $\mathcal{D}(\lambda, k)=0$ with $k \in \mathbb{R}$ is known as the "essential spectrum". Many readers who are unfamiliar with this term will nevertheless have calculated an essential spectrum, since this is how one derives the conditions for diffusion driven instability (Turing patterns).

When considering absolute stability, it is necessary to allow both $k$ and $\lambda$ to be complex-valued. For any given $\lambda$, we denote the roots for $k$ of the dispersion relation by $k_{1}(\lambda), k_{2}(\lambda), \ldots, k_{N}(\lambda)$, repeated with multiplicity, and labelled so that

$$
\operatorname{Im}\left(k_{1}\right) \leq \operatorname{Im}\left(k_{2}\right) \leq \cdots \leq \operatorname{Im}\left(k_{N}\right) .
$$

For absolute stability, one must consider a particular root $k_{n^{*}}$. A formal definition of $n^{*}$ is given in Sandstede and Scheel (2000b, Sect. 2.1), but it is most easily understood in the following intuitive way: for the PDE to be well defined on a finite domain with separated boundary conditions, $n^{*}$ boundary conditions are required at the righthand boundary, with $N-n^{*}$ conditions at the left-hand boundary. Thus, for (1a), (1b), $n^{*}=2$ (and $N=4$ ). As a different example, consider the model of Klausmeier (1999) for vegetation patterning on gentle slopes in semi-arid environments. This model involves equations for the plant biomass $m(x, t)$ and the water density $w(x, t)$, with the spatial coordinate $x$ increasing in the uphill direction:

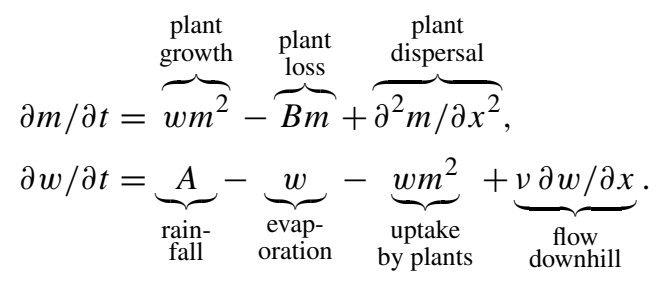

Here, the parameters $A, B$, and $v$ are all positive. For these equations, conditions on $m$ would be required at both boundaries, but a condition on $w$ is required only at the right-hand (upslope) boundary: Therefore, $n^{*}=2$ (and $N=3$ ).

The "absolute spectrum" is the set of values of $\lambda$ such that $\operatorname{Im} k_{n^{*}}=\operatorname{Im} k_{n^{*}+1}$. It is also useful to have a name for the more general set of $\lambda$ 's for which $\operatorname{Im} k_{i}=\operatorname{Im} k_{i+1}$ for any $i$, and this is known as the "generalised absolute spectrum". This latter set of eigenvalues was considered (without the name) in the original monograph of Briggs (1964), but the special significance of the case $i=n^{*}$ was not realised until Sandstede and Scheel's (2000b) work. Two final pieces of terminology are also useful: values of $\lambda$ for which $k_{n}=k_{n+1}$ are known as "branch points of index $n$ ", and "branch points in the absolute spectrum" are simply those with index $n^{*}$. Figure 6 shows examples of absolute spectra and generalised absolute spectra. Panels (a) and (b) show the absolute spectrum and the generalised absolute spectrum for the parameters used in Fig. 3b, c.

Note that the absolute spectrum differs from the essential spectrum because the latter corresponds to both perturbations that grow pointwise and perturbations that only grow while simultaneously moving. Thus, the absolute spectrum lies to the left 

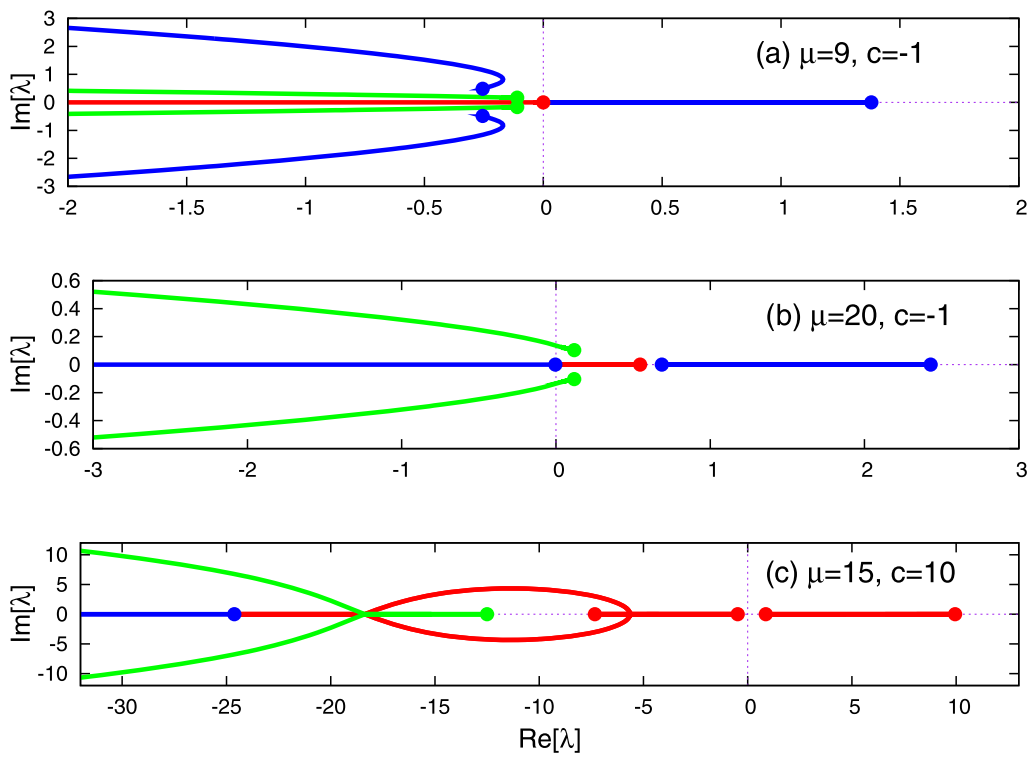

Fig. 6 Illustrations of the absolute spectrum (solid black; green online) and generalised absolute spectrum (all three line types/colours) for the Rosenzweig-MacArthur model (1a), (1b). Branch points are indicated by filled circles. The values of $\mu$ were (a) $\mu=9, c=-1$, (b) $\mu=20, c=-1$, (c) $\mu=15, c=10$; the other parameters were $a=1.3, b=4, d=2$. The numerical method was as described in the text: We first calculated the branch points (see Appendix A.1), then used these as starting points for the calculation of the generalised absolute spectrum, monitoring all of the $k_{i}$ 's during the continuation in order to determine which sections constitute the absolute spectrum. The dashed black curves (red online) correspond to $\operatorname{Im} k_{1}=\operatorname{Im} k_{2}$, while on the solid grey curves (blue online) $\operatorname{Im} k_{3}=\operatorname{Im} k_{4}$. The parameters used on panels (a) and (b) are the same as in the case of Fig. 3b and Fig. 3c. Note that the absolute spectrum can emanate not only from branch points but also from triple points, which are defined by $\operatorname{Im} k_{i}=\operatorname{Im} k_{i+1}=\operatorname{Im} k_{i+2}$ for some $i$. For example on panel (c), a triple point occurs at $\lambda=-18.37$ where the corresponding spatial eigenvalues are characterised by $\operatorname{Im} k_{1}=\operatorname{Im} k_{2}=\operatorname{Im} k_{3}$; parts of the absolute spectrum emanate from this triple point

of the essential spectrum in the eigenvalue complex plane; this is proved formally in Rademacher (2006).

For a spatially homogeneous steady state that is unstable as a solution of the kinetic ODEs, Sandstede and Scheel (2000b) showed that the absolute spectrum determines the nature of its instability in the PDEs as follows:

- the steady state is absolutely/convectively unstable according to whether there are/are not branch points of the absolute spectrum in the right-hand half of the complex plane;

- the steady state is remnantly/transiently unstable according to whether the absolute spectrum does/does not extend into the right-hand half of the complex plane.

More precise versions of these statements, including the required technical assumptions, are given in Sandstede and Scheel (2000b). Note that the "pinching condition" of Briggs (1964) translates to the condition that $\lambda$ is a branch point in the absolute spectrum. As mentioned at the end of Sect. 2, in all mathematical biology examples that have been considered to date, the most right-hand part of the absolute spectrum 
is a branch point, so that the notions of absolute and remnant instability, and also of convective and transient instability, coincide.

The relationship between the condition $\operatorname{Im} k_{n^{*}}=\operatorname{Im} k_{n^{*}+1}$ and these different types of instability is far from obvious. To motivate it intuitively, we present a simple example, due originally to Worledge et al. (1997, Sect. 3). We consider a system of two coupled reaction-diffusion equations

$$
\begin{aligned}
& u_{t}=u_{x x}+f(u, v), \\
& v_{t}=v_{x x}+g(u, v)
\end{aligned}
$$

on $-\ell<x<\ell$, subject to boundary conditions $u=v=0$ at $x= \pm \ell$, with $\ell$ large. Here, subscripts $x$ and $t$ denote partial derivatives. For these equations, $n^{*}=2$ and $N=4$. We denote by $\left(u_{s}, v_{s}\right)$ the homogeneous steady state whose stability is being considered, and write $\tilde{u}=u-u_{s}, \tilde{v}=v-v_{s}$. Then to leading order for small perturbations $\tilde{u}, \tilde{v}$,

$$
\begin{gathered}
\tilde{u}_{t}=\tilde{u}_{x x}+f_{u}\left(u_{s}, v_{s}\right) \tilde{u}+f_{v}\left(u_{s}, v_{s}\right) \tilde{v}, \\
\tilde{v}_{t}=\tilde{v}_{x x}+g_{u}\left(u_{s}, v_{s}\right) \tilde{u}+g_{v}\left(u_{s}, v_{s}\right) \tilde{v},
\end{gathered}
$$

where subscripts $u$ and $v$ denote partial derivatives. For a given value of the temporal eigenvalue $\lambda$, this has the general solution

$$
(\tilde{u}, \tilde{v})=e^{\lambda t} \sum_{j=1}^{4}\left(\bar{u}_{j}, \bar{v}_{j}\right) H_{j} e^{i k_{j} x},
$$

where $\left(\bar{u}_{j}, \bar{v}_{j}\right)$ is the eigenvector corresponding to the spatial eigenvalue $k_{j}$, and the $H_{j}$ 's are constants. Suppose first that the $\operatorname{Im} k_{j}$ 's are distinct. Then the behaviour of the solution at large positive $x$ is dominated by indices $j=1$ and $j=2$ in the summation, so that to leading order for large $\ell$, the boundary condition at $x=+\ell$ requires

$$
\sum_{j=1}^{2}\left(\bar{u}_{j}, \bar{v}_{j}\right) H_{j} e^{i k_{j} \ell}=(0,0) .
$$

Similarly, the boundary condition at $x=-\ell$ requires

$$
\sum_{j=3}^{4}\left(\bar{u}_{j}, \bar{v}_{j}\right) H_{j} e^{-i k_{j} \ell}=(0,0)
$$

to leading order. In general, (4) and (5) do not have any non-trivial solutions for the $H_{j}$ 's. This argument is unaffected if $\operatorname{Im} k_{1}=\operatorname{Im} k_{2}$ and/or $\operatorname{Im} k_{3}=\operatorname{Im} k_{4}$. However, if $\operatorname{Im} k_{2}=\operatorname{Im} k_{3}$ the situation changes. Then we have

$$
\sum_{j=1}^{3}\left(\bar{u}_{j}, \bar{v}_{j}\right) H_{j} e^{i k_{j} \ell}=\sum_{j=2}^{4}\left(\bar{u}_{j}, \bar{v}_{j}\right) H_{j} e^{-i k_{j} \ell}=(0,0)
$$


to leading order, which typically does admit non-trivial solutions for the $H_{j}$ 's. Hence, $\operatorname{Im} k_{2}=\operatorname{Im} k_{3}$ is the condition for non-trivial solutions of the linearised equations (3a). Therefore, the extension of the absolute spectrum into the right-hand half of the complex plane (i.e. a remnant instability) corresponds to the existence of such non-trivial solutions for $\lambda>0$, and this is exactly the condition for the steady state to be unstable on this bounded domain.

Branch points are the key to numerical calculation of the absolute spectrum. Since $\mathcal{D}$ has a repeated root for $k$ at a branch point, we have

$$
\mathcal{D}(\lambda, k)=0 \quad \text { and } \quad(\partial / \partial k) \mathcal{D}(\lambda, k)=0 .
$$

Now $\mathcal{D}$ is a polynomial in both $\lambda$ and $k$, and thus it is usually relatively straightforward to solve (6) for the branch points. For example, for (1a), (1b) one can eliminate $\lambda$ between the two equations in (6) to give a sixth order polynomial in $k$. This can be solved numerically and then each of the solutions can be substituted back into (6) to get the corresponding values of $\lambda$ (see Appendix A.1 for further details).

Branch points will be in the absolute spectrum if their index is $n^{*}$. To check this for a given branch point, one substitutes the calculated value of $\lambda$ back into $\mathcal{D}(\lambda, k)=0$ and solves the resulting polynomial in $k$. Two of the roots will be the repeated pair corresponding to the branch point. If these are $k_{n^{*}}$ and $k_{n^{*}+1}$, then the branch point is in the absolute spectrum; otherwise it is not.

The above procedure may seem a little involved at first sight, but in practice it is very straightforward and very quick, involving just the numerical solution of polynomials. In the Appendix, we demonstrate the calculation of the branch points for the Rosenzweig-MacArthur (1963) model (1a), (1b) and the Klausmeier (1999) model (2a), (2b).

Having calculated the branch points, one can proceed to calculate the entire generalised absolute spectrum via numerical continuation. Using a branch point of in$\operatorname{dex} j$ as a starting point, one performs a numerical continuation of the polynomial $\mathcal{D}(\lambda, k)$, using $\operatorname{Re} k_{j}-\operatorname{Re} k_{j+1}$ as a continuation variable. This method was proposed by Rademacher et al. (2007), and it is also described in detail in Smith et al. (2009); the latter paper includes an online supplement with a detailed tutorial guide and sample code. Repeating this procedure for each branch point generates the entire generalised absolute spectrum, since for constant coefficient problems all curves of generalised absolute spectrum emanate from a branch point, at least for a wide class of equations including reaction-diffusion systems (Rademacher et al. 2007). The same statement does not necessarily hold for the absolute spectrum itself ${ }^{1}$ and, therefore, it is not possible to calculate the absolute spectrum directly using this approach. Rather it is necessary to calculate the generalised absolute spectrum, and to monitor all four roots for $k$ of $D(\lambda, k)=0$ as one moves along it. The absolute spectrum is simply the part of the generalised absolute spectrum for which $\operatorname{Im} k_{n^{*}}=\operatorname{Im} k_{n^{*}+1}$.

In practice, one is interested in the most unstable point in the absolute spectrum, and also its most unstable branch point. Usually these are the same: This will

\footnotetext{
${ }^{1}$ Instead, curves of absolute spectrum can emanate from "triple points", defined by $\operatorname{Im} k_{i}=\operatorname{Im} k_{i+1}=$ $\operatorname{Im} k_{i+2}$ for some $i$ (Rademacher et al. 2007; Smith et al. 2009). See Fig. 6c for an example of parts of an absolute spectrum emanating from triple points.
} 
Fig. 7 An example of stability boundaries in the $(\mu, c)$ plane for (1a), (1b). The vertical line (dot-dash; solid red online) marks the transition between the coexistence steady state being stable and unstable, while the solid curve (blue online) shows the transition between convective and absolute instability. The latter curve was plotted via the calculation of branch points, as described in Appendix A.1. The parameter values were $a=1.3, b=4$, $d=2$

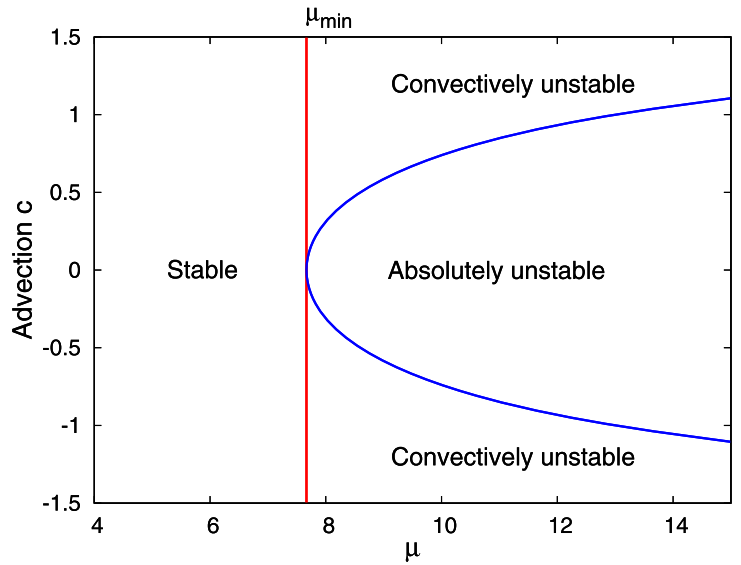

be the case unless there is a non-transient convective instability (see Sect. 2). For some systems, it has been proved that the most unstable point in the absolute spectrum is a branch point (Smith et al. 2009), but the complexity of most models of biological phenomena puts them outside the compass of these results. Therefore, when considering absolute stability in a model for the first time, it is good practice to calculate the full absolute spectrum for a selection of parameter sets; usually these will show that the most unstable point in the absolute spectrum is a branch point in each case. One can then have confidence in making statements about absolute/convective/remnant/transient stability on the basis of branch points, which is usually a very simple calculation. In fact, in the physics literature, where different types of instability are often considered, many authors draw their conclusions based only on calculations of branch points. As an example of a calculation done in this way, Fig. 7 shows the boundary between convective and absolute stability of the coexistence steady state in the $\mu-c$ plane for (1a), (1b), based simply on numerically solving a polynomial to determine the branch points, as described in Appendix A.1.

\section{Quantitative Calculations Using Absolute Stability}

The example in Sect. 3 illustrates how determination of the type of instability can be used to understand and predict important qualitative features of spatiotemporal behaviour. However, the theory is in fact much more powerful than this. Quantitative information can also be obtained, by investigating stability in different frames of reference. We will demonstrate this for the solutions presented in Sect. 3, by adapting the method proposed by Sherratt et al. (2009) for invasions in reaction-diffusion systems of $\lambda-\omega$ type.

Given a PDE with space variable $x$ and time variable $t$, one changes to a moving frame of reference in the usual way, by changing coordinates to $z=x-V t$ and $t$. The frame velocity $V$ is an additional parameter. For any given $V$, one can use the methods in Sect. 5 to calculate the most unstable point in the absolute spectrum, say 


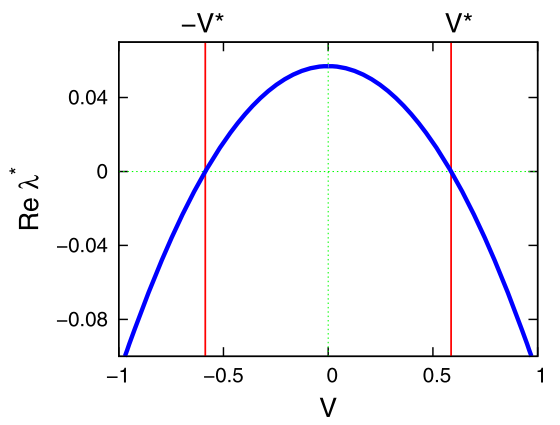

Fig. 8 An example of the dependence of $\operatorname{Re} \lambda^{*}$ against $V$. For a given velocity $V, \lambda^{*}(V)$ is the most unstable point in the absolute spectrum, meaning that $\operatorname{Re} \lambda^{*}(V)$ is the maximum growth rate of perturbations moving with velocity $V$. The example shown is for the Rosenzweig and MacArthur (1963) model (1a), (1b) with parameters $a=1.3, b=4, c=0, d=2, \mu=9$. Calculation of the absolute spectrum for this case, with a variety of different $V$ values, showed that the most unstable point in the absolute spectrum was a branch point in all cases. Therefore, we determined $\lambda^{*}(V)$ by calculating branch points, as described in Appendix A.1

$\lambda^{*}(V)$. This is the eigenvalue associated with the most unstable linear mode moving with velocity $V$. We denote by $k^{*}$ the corresponding value of $k$. Figure 8 shows a typical plot of $\operatorname{Re} \lambda^{*}$ against $V$, for the model (1a), (1b). There is a finite range of velocities for which there are growing linear modes: all linear modes moving with speed $(=|V|)$ greater than $V^{*}$ are decaying.

A plot such as Fig. 8 is quite instructive in its own right. For example, we presented simulations in Sect. 1 showing that there can be a plateau region behind the invasion front in which the (unstable) co-existence steady state is "dynamically stabilised". Denoting the invasion velocity by $V_{\text {front }}$, the condition for such a plateau is $V_{\text {front }}>V^{*}$, so that the invasion can outrun all growing linear modes (Dagbovie and Sherratt 2013). But the curve $\lambda^{*}(V)$ can also form the basis of calculations. For example, a natural question arising from the simulations shown in Sect. 3 is how the width of the steady state region depends on parameters. To determine this, note that the amplitude of a linear mode travelling with velocity $V$ will double over the time period $\log (2) / \operatorname{Re} \lambda^{*}(V)$. During this time, the linear mode moves a distance $V \log (2) / \operatorname{Re} \lambda^{*}(V)$ while the transition front moves a distance $V_{\text {front }} \log (2) / \operatorname{Re} \lambda^{*}(V)$. Recall that $V_{\text {front }}$ is the speed of the front, which can be found using the theory of minimal spreading speeds (van Saarloos 2003); in the absence of advection, this speed is given by $V_{\text {front } 0}=2 \sqrt{\frac{d}{a b}\left(\frac{a \mu}{\mu+1}-1\right)}$ but in the context of this paper, it is defined by $V_{\text {front }}=-V_{\text {front } 0}-c$. Therefore, the linear mode doubles in amplitude while moving a distance $\left(V-V_{\text {front }}\right) \log (2) / \operatorname{Re} \lambda^{*}(V)$ from the front. One can reasonably assume that the transition front applies a perturbation to the co-existence steady state that contains all unstable linear modes. Therefore, the perturbation doubles in amplitude over the "doubling distance"

$$
x_{d b l}=\log (2)\left(V_{d b l}-V_{\text {front }}\right) / \operatorname{Re} \lambda^{*}\left(V_{d b l}\right)
$$



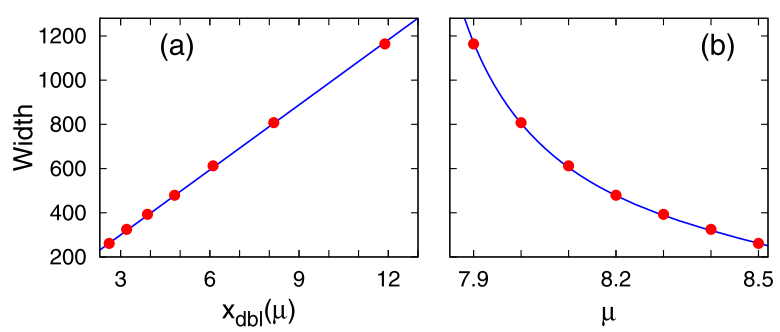

Fig. 9 An illustration of the correlation between the doubling distance $x_{d b l}$ and the width of the steady state region in simulations of (1a), (1b) with the zero flux condition $h_{x}+c h=d p_{x}+c p=0$ at the left-hand boundary and the Danckwert condition $h_{x}=p_{x}=0$ at the right-hand boundary, as in Fig. 5. The parameter $\mu$ was varied between 7.9 and 8.5. (a) A plot of $x_{d b l}$ against the numerically calculated width (dots), showing a very strong linear correlation between the two quantities (regression coefficient $=0.999$ ). This confirms that the dependence on parameters of the width of the steady state region is captured by $x_{d b l}$. The line is the best-fit regression line, which has slope 98.05 and intercept 5.795; there is a non-zero intercept because our method for measuring the width of the steady-state region excludes small portions on either side (see Smith and Sherratt 2009 for further discussion). (b) A plot of the width of the steady state region (dots) against $\mu$. We superimpose on this a curve showing $x_{d b l}$, rescaled using the regression line found from (a). The doubling distance was calculated using (7) and (8). The width of the steady state region was calculated using numerical simulations performed as in Fig. 5; the space and time discretisations are as in Fig. 1. The method used to measure the width in numerical simulations is described in Dagbovie and Sherratt (2013). The parameter values were $a=1.3, b=4, c=-0.5, d=2$

where $V_{d l b}$ minimises $\left(V-V_{\text {front }}\right) / \operatorname{Re} \lambda^{*}(V)$, i.e.

$$
\left.\left(V_{d b l}-V_{\text {front }}\right) \frac{d}{d V} \operatorname{Re} \lambda^{*}(V)\right|_{V=V_{d b l}}=\operatorname{Re} \lambda^{*}\left(V_{d b l}\right) .
$$

Numerical solution of (8) is made relatively straightforward by the identity $\left.(d / d V) \operatorname{Re} \lambda^{*}(V)\right|_{V=V_{d b l}}=-\operatorname{Im} k^{*}\left(V_{d b l}\right)$ (Sherratt et al. 2009). In some cases, (8) will have more than one solution: Then the relevant solution is the one giving the smallest value of $x_{d b l}$; an example of this is given in Fig. 5 of Smith and Sherratt (2009). The formula (7) for $x_{d b l}$ contains all of the parameter dependence of the width of the steady state region in the simulations shown in Sect. 3. For example, Fig. 9 plots $x_{d b l}$ against estimates of the width from simulations as the parameter $\mu$ is varied, demonstrating their linear relationship.

\section{Summary and Discussion}

Biology abounds with complex spatiotemporal phenomena. Mathematical models have been highly successful at reproducing this complexity, but currently our ability to numerically simulate such phenomena far outstrips our ability to provide an underlying mathematical understanding. As a result, models can sometimes fail to fulfil their potential for qualitative and quantitative prediction. The theory of absolute stability provides a tool that has the potential to redress this inbalance in some cases, and whose use within mathematical biology is currently almost non-existent. Until recently, one had to overcome a steep learning curve to make practical use of this theory, but new computational methods based on the absolute spectrum now make it 
easily accessible to non-specialists. We hope that this article will encourage greater use of these methods within the mathematical biology community.

We have focussed on the stability of spatially homogeneous solutions, for which both the theory and methods for numerical calculation are most complete. The absolute stability of spatially varying solutions was first considered by Brevdo and Bridges (1996, 1997b). Most of the key concepts that we have discussed extend from homogeneous solutions to a wide variety of non-uniform solutions, including solutions of reaction-diffusion systems that approach either constant or spatially periodic states at infinity. One complication is that the absolute spectrum can be augmented by discrete eigenvalues, and Wheeler and Barkley (2006) discuss an example of spiral wave break-up in which absolute stability is determined by discrete eigenvalues rather than by the absolute spectrum. However, for large classes of systems it can be shown there are no such discrete eigenvalues: this includes spatially homogeneous solutions, and wavetrain solutions of reaction-diffusion systems (Sandstede 2002, Sect. 3.4.2).

The biggest difficulty when considering spatially varying solutions is numerical calculation. Numerical continuation is still a viable method for calculation of the absolute spectrum (Rademacher et al. 2007), but finding appropriate starting points is problematic. In particular, branch points lose their suitability because for spatially varying solutions there are in general infinitely many branch points (Rademacher et al. 2007, Sect. 4.3). Alternative approaches to the generation of starting points are discussed in Sect. 4.4 of Rademacher et al. (2007), but in most cases the only systematic way of calculating absolute stability for non-constant solutions is the method of Brevdo and co-workers (Brevdo 1995; Brevdo et al. 1999; see also Suslov 2006).

The restriction to spatially uniform solutions is more inclusive than it appears superficially. For example, wavetrain solutions to reaction-diffusion systems of " $\lambda-\omega$ type" are spatially uniform when the equations are written in phase-amplitude form (Kopell and Howard 1973), and the same is true for wavetrains in the complex Ginzburg-Landau equation (Aranson and Kramer 2002). Also, the absolute spectrum of wave fronts and pulses in reaction-diffusion systems depends only on the asymptotic states behind and ahead of the wave (Sandstede 2002, Sects. 3.4.3, 3.4.4), although in this case one will also need to consider discrete eigenvalues, and these do depend on the wave profile.

We also emphasise that our remarks only concern behaviour in one space dimension. Absolute stability has been widely used to study spiral wave break-up (e.g. Aranson et al. 1992; Sandstede and Scheel 2000a; Wheeler and Barkley 2006), but this is effectively a one-dimensional problem. There has been some discussion of absolute stability for genuinely two-dimensional solutions in the hydrodynamics literature (e.g. Chomaz 2004; Biancofiore et al. 2011), but a detailed theory is currently lacking. This, and more effective numerical methods for spatially varying solutions, are major current objectives for the research community working on absolute stability.

In ecology, the term 'stability' plays a central role. In their 1997 review of the ecological literature, Grimm and Wissel (1997) found 163 definitions of 70 different stability concepts. Yet, the theory of absolute stability has been, to our knowledge, absent from the ecological literature, and it appears that the spatial dimension is generally lacking in ecological stability concepts. 
This is particularly surprising since stream ecologists (and water resource managers alike) have long been on the quest of how lotic systems respond to disturbances and spatial variabilities. Much of the current knowledge comes from experiments in study sites that comprise only a small fragment of the stream or river (Fausch et al. 2002). But how do populations much further downstream, i.e. outside the study arena, respond to upstream perturbations (Cooper et al. 1998)? This is a key question in the assessment of instream flow needs, or how the location of wastewater treatment plants affects environmental conditions downstream (Anderson et al. 2006). One attempt to address this question is the concept of the response length (Anderson et al. 2005), a characteristic length scale measuring the scale over which environmental disturbances are felt by distant populations.

Convective instabilities appear particularly intriguing in this context. The perturbed steady state remains stable locally, but is enormously brought out of equilibrium further downstream (cf. Fig. 5b). Small-scale experiments and observations might only see the local (stable) response, but miss out on the instabilities arising further away. In Sect. 6, we have shown how the width of the region where the steady state remains stable can be calculated quantitatively (see also Dagbovie and Sherratt 2013). This may be useful information for the design of experiments, ecological monitoring as well as environmental assessment.

The convective and absolute instabilities that we have described do not necessarily need to be 'harmful' to the populations. On the contrary, the spatiotemporal oscillations caused by the instabilities can be beneficial. For example, they might facilitate non-equilibrium coexistence of species that would otherwise mutually exclude each other (Armstrong and McGehee 1980; Huisman and Weissing 1999). In a more water-flow oriented context, Scheuring et al. (2000) have shown that the chaotic flow around a cylindrical obstacle creates a small-scale mosaic ensuring that coexisting species can co-exist. Similarly, Lee (2012) has argued that rotational flow, as caused by a rock, can increase survival probabilities of predators and prey. Petrovskii et al. (2004) have shown, in a non-advective model that spatiotemporal chaos can reverse the paradox of enrichment, and thus make the persistence of predators and prey more likely.

Convective and absolute instabilities can lead to spatial and spatiotemporal patterns. Many of the well-known mechanisms inducing biological pattern formation are based on the phenomenon that steady states which are locally stable in the kinetic ODEs become destabilised in the PDEs. For instance, the diffusion-driven instabilities of the Turing mechanism are caused by the addition of significantly different diffusivities, which lead to short-range activation and long-range inhibition. Advective environments feature a number of spatiotemporal pattern formation mechanisms that cannot arise in non-advective systems. In contrast to the Turing mechanism, they do not require activator-inhibitor type of interactions, but they critically depend on other conditions, e.g. a significant difference in the flow experienced by species (Rovinsky and Menzinger 1992; Perumpanani et al. 1995; Malchow 2000) or resource-dependent dispersal (Anderson et al. 2012). However, they all have in common that the steady states in the kinetic ODEs are locally stable.

The patterns generated by convective and absolute instabilities do not require any of these conditions (activator-inhibitor dynamics; significantly different mobilities 
of the interacting species; resource-dependent dispersal). However, in contrast to the previously mentioned mechanisms, the steady states are already unstable in the kinetic ODEs. Considering the abundance of endogenous population oscillations, this is frequently the case in nature and can be caused by a number of mechanisms (see Turchin 2003). Hence, convective and absolute instabilities appear to be a fairly general mechanism for spatiotemporal pattern formation in ecology.

Streams and river are particularly iconic examples of flow-dominated systems, but there are many others. Marine organisms dispersed in longshore currents (Gaylord and Gaines 2000) or plant seeds and insects dispersed by winds with a prevailing wind direction (Levine 2003) are also environments with a predominantly unidirectional flow. These systems are strongly characterised by the importance of longitudinal transport through habitat. The theory of absolute stability appears to have a lot to offer when there is a downstream bias that can induce very different spatial responses to localised perturbations.

Acknowledgements J.A.S. acknowledges discussions with Leonid Brevdo (Louis Pasteur University, Strasbourg), Jens Rademacher (CWI, Amsterdam), Björn Sandstede (Brown University) and Matthew Smith (Microsoft Research, Cambridge). A.S.D. was supported by the Centre for Analysis and Nonlinear PDEs funded by the UK EPSRC grant EP/E03635X and the Scottish Funding Council. F.M.H. acknowledges discussions with Mark Lewis (University of Alberta), Sergei Petrovskii (University of Leicester) and Frithjof Lutscher (University of Ottawa).

\section{Appendix: Examples of Calculating Branch Points}

In this Appendix, we show how to calculate the branch points for the RosenzweigMacArthur model (1a), (1b) and the Klausmeier model (2a), (2b). We present these calculations in some detail, with the aim of providing templates that readers can follow when performing corresponding calculations for their own models.

\section{A.1 Branch Points for the Rosenzweig-MacArthur Model}

Recall that the Rosenzweig-MacArthur model (1a), (1b) has a unique homogeneous co-existence steady state $\left(h_{s}, p_{s}\right)$ where $h_{s}=1 /(a \mu-\mu)$ and $p_{s}=\left(1-h_{s}\right)(1+$ $\left.\mu h_{s}\right) / \mu$. We begin by linearising (1a), (1b) about $\left(h_{s}, p_{s}\right)$ giving

$$
\begin{aligned}
& \tilde{p}_{t}=\alpha \tilde{p}+\beta \tilde{h}+c \tilde{p}_{x}+d \tilde{p}_{x x}, \\
& \tilde{h}_{t}=\gamma \tilde{p}+\delta \tilde{h}+c \tilde{h}_{x}+\tilde{h}_{x x},
\end{aligned}
$$

where $\tilde{p}=p-p_{s}, \tilde{h}=h-h_{s}$, and $\alpha, \beta, \gamma, \delta$ are coefficients from linearisation and are given by

$$
\begin{aligned}
& \alpha=\frac{\mu h_{s}}{b\left(1+\mu h_{s}\right)}-\frac{1}{a b}, \\
& \beta=\frac{\mu p_{s}}{b\left(1+\mu h_{s}\right)^{2}}, \\
& \gamma=1-2 h_{s}-\frac{\mu p_{s}}{\left(1+\mu h_{s}\right)^{2}},
\end{aligned}
$$




$$
\delta=-\frac{\mu h_{s}}{1+\mu h_{s}} .
$$

Substituting $(\tilde{p}, \tilde{h})=(\bar{p}, \bar{h}) \exp (i k x+\lambda t)$ into (1a), (1b) and requiring $\bar{p}$ and $\bar{h}$ to be non-zero gives the dispersion relation

$$
\begin{aligned}
\mathcal{D}(\lambda, k)= & d k^{4}-c i k^{3}(d+1)-k^{2}\left(\alpha-\lambda+d \gamma-d \lambda+c^{2}\right) \\
& +c i k(\gamma+\alpha-2 \lambda)+(\alpha-\lambda)(\gamma-\lambda)-\delta \beta=0 .
\end{aligned}
$$

Branch points are double roots of the dispersion relation for $k$, and satisfy (9) and also

$$
\begin{aligned}
0 & =\partial \mathcal{D} / \partial k \\
& =4 d k^{3}-3 k^{2}(1+d) c i-2 k(\gamma+d \alpha-(1+d) \lambda)+c^{2}+c i(\alpha+\gamma-2 \lambda) \Rightarrow \\
\lambda & =\frac{4 d k^{3}-3 c i d k^{2}-2 d k \gamma+\alpha c i-2 c^{2} k-3 c i k^{2}-2 \alpha k+c i \gamma}{-2(k+d k-c i)} .
\end{aligned}
$$

Substituting (10) into (9) gives the following hexic polynomial in $k$ :

$$
\begin{aligned}
& {\left[-4 d(d-1)^{2}\right] k^{6}+\left[2 c i(d+1)(d-1)^{2}\right] k^{5}+\left[(d-1)\left(c^{2} d+8 d \alpha-8 d \gamma-c^{2}\right)\right] k^{4}} \\
& \quad+[4 c i(-\alpha+\gamma)(d-1)(d+1)] k^{3} \\
& \quad+\left[2 c^{2}(d-1)(\gamma-\alpha)-4 \beta \delta(d+1)^{2}-4 d(\alpha-\gamma)^{2}\right] k^{2} \\
& \quad+\left[2 i\left(4 \beta \delta+\alpha^{2}-2 \alpha \gamma+\gamma^{2}\right) c(d+1)\right] k+c^{2}\left(4 \beta \delta+\alpha^{2}-2 \alpha \gamma+\gamma^{2}\right)=0 .
\end{aligned}
$$

We must now proceed numerically and we fix $a=1.3, b=4.0, c=-1, d=2$, and $\mu=9$. These parameter values satisfy $\mu>\mu_{\text {crit }}$, so that the coexistence steady state is unstable. Substituting these values into (11), we obtain six roots for $k$, two real and two pairs of complex conjugates. We then substitute each into (10) to find the corresponding value of $\lambda$. To determine whether these branch points belong to the absolute spectrum, we substitute each $\lambda$ value into (9) and solve for $k$, giving the repeated roots found from (11) and two others.

Branch point $k=0.676 i$. Substituting this value of $k$ into (10) gives $\lambda=1.380$. Substituting this value of $\lambda$ back into (9) gives a quartic polynomial for $k$ whose roots are $-1.727 i,-1.125 i, 0.676 i, 0.676 i$. Recall that a branch point is in the absolute spectrum if the repeated roots are $k_{2}$ and $k_{3}$, when the roots $k_{1}, k_{2}, k_{3}$ and $k_{4}$ of (9) are labelled in increasing order of their imaginary parts. In this case, the repeated roots are $k_{3}$ and $k_{4}$ so that the branch point is not in the absolute spectrum.

Branch point $k=-0.473-0.013 i$. Substituting this value of $k$ into (10) gives $\lambda=$ $-0.255+0.483 i$. Substituting this value of $\lambda$ back into (9) gives a quartic polynomial for $k$ whose roots are $0.570-0.943 i, 0.377-0.530 i,-0.473-0.013 i$, $-0.473-0.013 i$ Therefore, the repeated roots are $k_{3}$ and $k_{4}$ so that the branch point is not in the absolute spectrum. 
Branch point $k=0.473-0.013 i$. Substituting this value of $k$ into (10) gives $\lambda=$ $-0.255-0.483 i$. Substituting this value of $\lambda$ back into (9) gives a quartic polynomial for $k$ whose roots are $-0.570-0.943 i,-0.377-0.530 i, 0.473-0.013 i$, $0.473-0.013 i$. Therefore, the repeated roots are $k_{3}$ and $k_{4}$ so that the branch point is not in the absolute spectrum.

Branch point $k=0.001-0.334 i$. Substituting this value of $k$ into (10) gives $\lambda=$ $-0.110-0.167 i$. Substituting this value of $\lambda$ back into (9) gives a quartic polynomial for $k$ whose roots are $-0.314-0.816 i, 0.001-0.334 i, 0.001-0.334 i$, $0.312-0.0165 i$. Therefore, the repeated roots are $k_{2}$ and $k_{3}$ so that the branch point is in the absolute spectrum.

Branch point $k=-0.001-0.334 i$. Substituting this value of $k$ into (10) gives $\lambda=$ $-0.110+0.167 i$. Substituting this value of $\lambda$ back into (9) gives a quartic polynomial for $k$ whose roots are $0.314-0.816 i,-0.001-0.334 i,-0.001-0.334 i$, $-0.312-0.0165 i$. Therefore, the repeated roots are $k_{2}$ and $k_{3}$ so that the branch point is in the absolute spectrum.

Branch point $k=-0.732 i$. Substituting this value of $k$ into (10) gives $\lambda=0.000$.

Substituting this value of $\lambda$ back into (9) gives a quartic polynomial for $k$ whose roots are $-0.732 i,-0.732 i, 0.160-0.017 i,-0.160-0.017 i$. Therefore, the repeated roots are $k_{1}$ and $k_{2}$ so that the branch point is not in the absolute spectrum.

Therefore, of the six branch points, two are in the absolute spectrum, with the corresponding eigenvalues being $-0.110 \pm 0.167 i$. Since these eigenvalues have negative real parts, the steady state $\left(h_{s}, p_{s}\right)$ is absolutely stable. To determine whether the convective instability is of transient or remnant type, it is necessary to calculate the absolute spectrum. This can be done via numerical continuation of the generalised absolute spectrum, using the six branch points listed above as starting points, as discussed in Sect. 5. This shows that the branch points are the most unstable points in the absolute spectrum, so that the steady state has a transient convective instability.

\section{A.2 Branch Points for the Klausmeier Model}

For all parameters, the Klausmeier model (2a), (2b) has a "desert" steady state $m=0$, $w=A$. When $A \geq 2 B$, there are two further steady states $\left(m_{ \pm}, w_{ \pm}\right)$where

$$
m_{ \pm}=\frac{2 B}{A \pm \sqrt{A^{2}-4 B^{2}}}, \quad w_{ \pm}=\frac{A \pm \sqrt{A^{2}-4 B^{2}}}{2} .
$$

Ecologically realistic values of $B$ are relatively small, and in particular satisfy $B<2$ (Klausmeier 1999; Rietkerk et al. 2002). Under this constraint, $\left(m_{-}, w_{-}\right)$is stable as a solution of the kinetics ODEs, although it can be destabilised by the diffusion and advection terms, leading to spatial patterns (Klausmeier 1999; Sherratt 2005, 2010). However, $\left(m_{+}, w_{+}\right)$is unstable as a solution of the kinetic ODEs, and we will consider the nature of its instability as a solution of the PDEs (2a), (2b).

We begin by linearising $(2 \mathrm{a}),(2 \mathrm{~b})$ about $\left(m_{+}, w_{+}\right)$, giving

$$
\begin{aligned}
& \tilde{m}_{t}=\tilde{\alpha} \tilde{m}+\tilde{\beta} \tilde{w}+\tilde{m}_{x x}, \\
& \tilde{w}_{t}=\tilde{\gamma} \tilde{m}+\tilde{\delta} \tilde{w}+v \tilde{w}_{x},
\end{aligned}
$$


where $\tilde{m}=m-m_{+}, \tilde{w}=w-w_{+}$, and the linear coefficients $\alpha, \beta, \gamma, \delta$ are given by

$$
\begin{aligned}
& \tilde{\alpha}=B, \\
& \tilde{\beta}=\frac{A-\sqrt{A^{2}-4 B^{2}}}{A+\sqrt{A^{2}-4 B^{2}}}, \\
& \tilde{\gamma}=-2 B, \\
& \tilde{\delta}=\frac{-2 A}{A+\sqrt{A^{2}-4 B^{2}}} .
\end{aligned}
$$

Substituting $(\tilde{m}, \tilde{w})=(\bar{m}, \bar{w}) \exp (i k x+\lambda t)$ into (12a), (12b) and requiring $\bar{m}$ and $\bar{w}$ to be non-zero gives the dispersion relation

$$
\tilde{\mathcal{D}}(\lambda, k)=\lambda^{2}+\lambda\left(k^{2}-\tilde{\alpha}-i k \nu-\tilde{\delta}\right)+\left(\tilde{\alpha}-k^{2}\right)(i k \nu+\tilde{\delta})-\tilde{\beta} \tilde{\gamma}=0 .
$$

Branch points are double roots (for $k$ ) of the dispersion relation, and satisfy (14) and also

$$
\begin{aligned}
& 0=\partial \tilde{\mathcal{D}} / \partial k=\lambda(2 k-i v)-\left(3 i k^{2} v+2 \tilde{\delta} k-i v \tilde{\alpha}\right) \Rightarrow \\
& \lambda=\left(3 i k^{2} v+2 \tilde{\delta} k-i v \tilde{\alpha}\right) /(2 k-i v) .
\end{aligned}
$$

Substituting (15) into (14) gives a quintic polynomial in $k$ :

$$
\begin{aligned}
& \left(3 i k^{2} v+2 \tilde{\delta} k-i v \tilde{\alpha}\right)^{2}+(2 k-i v)\left(3 i k^{2} v+2 \tilde{\delta} k-i v \tilde{\alpha}\right)\left(k^{2}-\tilde{\alpha}-i k v-\tilde{\delta}\right) \\
& \quad+(2 k-i v)^{2}\left[\left(\tilde{\alpha}-k^{2}\right)(i k v+\tilde{\delta})-\tilde{\beta} \tilde{\gamma}\right]=0
\end{aligned}
$$

We must now proceed numerically, and we will fix the parameter values to be $A=2, B=0.5$ and $v=20$. These parameters satisfy the condition $A>2 B$, but otherwise they are chosen arbitrarily. The value $v=20$ is too small for ecological realism: The formula for the dimensionless parameter $v$ involves the ratio of the advection rate of water and the (square root of the) plant diffusion coefficient (Klausmeier 1999; Sherratt 2005), so that $v$ is relatively large, with Klausmeier's (1999) estimate being 182.5. However, we use the smaller value to improve the clarity of the numerical calculations. Substituting the parameter values into (16) gives five distinct roots for $k$, two complex and three pure imaginary. For each, we substitute into (15) to find the corresponding value of $\lambda$. We then substitute this value of $\lambda$ into (14) to determine whether the branch point is in the absolute spectrum. We performed all of the various calculations using the software package MAPLE with 20 decimal places, but for clarity we give results to 3 decimal places.

Branch point $k=-0.099+i 0.012$. Substituting this value of $k$ into (15) gives $\lambda=$ $0.473+i 0.022$. Substituting this value of $\lambda$ back into (14) gives a cubic polynomial for $k$ whose roots are $-0.099+i 0.012,-0.099+i 0.012,0.199-i 0.101$. Recall from Sect. 5 that the branch point is in the absolute spectrum if the repeated roots are $k_{2}$ and $k_{3}$, when the roots $k_{1}, k_{2}, k_{3}$ of (14) are labelled in increasing order of their imaginary parts. Therefore, in this case the branch point is in the absolute spectrum. 
Branch point $k=-i 19.950$. Substituting this value of $k$ into (15) gives $\lambda=398.112$. Substituting this value of $\lambda$ back into (14) gives a cubic polynomial for $k$ whose roots are $-i 19.950,-i 19.950$ and $i 19.94$. Therefore, the repeated roots are $k_{1}$ and $k_{2}$, so that this branch point is not in the absolute spectrum.

Branch point $k=-i 19.892$. Substituting this value of $k$ into (15) gives $\lambda=396.587$. Substituting this value of $\lambda$ back into (14) gives a cubic polynomial for $k$ whose roots are $-i 19.892,-i 19.892$ and $i 19.902$. Therefore, the repeated roots are $k_{1}$ and $k_{2}$, so that this branch point is not in the absolute spectrum.

Branch point $k=-i 0.181$. Substituting this value of $k$ into (15) gives $\lambda=0.569$. Substituting this value of $\lambda$ back into (14) gives a cubic polynomial for $k$ whose roots are $-i 0.181,-i 0.181$, and $i 0.281$. Therefore, the repeated roots are $k_{1}$ and $k_{2}$, so that this branch point is not in the absolute spectrum.

Branch point $k=0.099+i 0.012$. Substituting this value of $k$ into (15) gives $\lambda=$ $0.473-i 0.022$. Substituting this value of $\lambda$ back into (14) gives a cubic polynomial for $k$ whose roots are $-0.199-i 0.101,0.099+i 0.012$, and $0.099+i 0.012$. Therefore, the repeated roots are $k_{2}$ and $k_{3}$, so that this branch point is in the absolute spectrum.

Therefore, of the five branch points, two are in the absolute spectrum, with the corresponding eigenvalues being $0.473 \pm i 0.022$. Since these eigenvalues have positive real part, the steady state $\left(m_{+}, w_{+}\right)$is absolutely unstable.

\section{References}

Anderson, K. E., Nisbet, R. M., Diehl, S., \& Cooper, S. D. (2005). Scaling population responses to spatial environmental variability in advection-dominated systems. Ecol. Lett., 8, 933-943.

Anderson, K. E., Paul, A. J., McCauley, E., Jackson, L. J., Post, J. R., \& Nisbet, R. M. (2006). Instream flow needs in streams and rivers: the importance of understanding ecological dynamics. Front. Ecol. Environ., 4, 309-318.

Anderson, K. E., Hilker, F. M., \& Nisbet, R. M. (2012). Directional dispersal and emigration behavior drive a flow-induced instability in a stream consumer-resource model. Ecol. Lett., 15, 209-217.

Aranson, I. S., \& Kramer, L. (2002). The world of the complex Ginzburg-Landau equation. Rev. Mod. Phys., 74, 99-143.

Aranson, I. S., Aranson, L., Kramer, L., \& Weber, A. (1992). Stability limits of spirals and traveling waves in nonequilibrium media. Phys. Rev. A, 46, R2992-R2995.

Armstrong, R. A., \& McGehee, R. (1980). Competitive exclusion. Am. Nat., 115, 151-170.

Beyn, W.-J., \& Lorenz, J. (1999). Stability of travelling waves: dichotomies and eigenvalue conditions on finite intervals. Numer. Funct. Anal. Optim., 20, 201-244.

Biancofiore, L., Gallaire, F., \& Pasquetti, R. (2011). Influence of confinement on a two-dimensional wake. J. Fluid Mech., 688, 297-320.

Brandt, M. J., \& Lambin, X. (2007). Movement patterns of a specialist predator, the weasel Mustela nivalis exploiting asynchronous cyclic field vole Microtus agrestis populations. Acta Theriol., 52, 13-25.

Brevdo, L. (1988). A study of absolute and convective instabilities with an application to the Eady model. Geophys. Astrophys. Fluid Dyn., 40, 1-92.

Brevdo, L. (1995). Convectively unstable wave packets in the Blasius boundary layer. Z. Angew. Math. Mech., 75, 423-436.

Brevdo, L., \& Bridges, T. J. (1996). Absolute and convective instabilities of spatially periodic flows. Philos. Trans. R. Soc. Lond. A, 354, 1027-1064.

Brevdo, L., \& Bridges, T. J. (1997a). Absolute and convective instabilities of temporally oscillating flows. Z. Angew. Math. Phys., 48, 290-309.

Brevdo, L., \& Bridges, T. J. (1997b). Local and global instabilities of spatially developing flows: cautionary examples. Proc. R. Soc. Lond. A, 453, 1345-1364. 
Brevdo, L., Laure, P., Dias, F., \& Bridges, T. J. (1999). Linear pulse structure and signalling in a film flow on an inclined plane. J. Fluid Mech., 396, 37-71.

Briggs, R. J. (1964). Electron-stream interaction with plasmas. Cambridge: MIT Press.

Chomaz, J. M. (2004). Transition to turbulence in open flows: what linear and fully nonlinear local and global theories tell us. Eur. J. Mech. B, 23, 385-399.

Chomaz, J. M. (2005). Global instabilities in spatially developing flows: non-normality and nonlinearity. Annu. Rev. Fluid Mech., 37, 357-392.

Cooper, S. D., Diehl, S., Kratz, K., \& Sarnelle, O. (1998). Implications of scale for patterns and processes in stream ecology. Aust. J. Ecol., 23, 27-40.

Dagbovie, A. S., \& Sherratt, J. A. (2013, accepted). Absolute stability and dynamical stabilisation in predator-prey systems. J. Math. Biol. doi:10.1007/s00285-013-0672-8.

Dunbar, S. R. (1986). Traveling waves in diffusive predator-prey equations-periodic orbits and point-toperiodic heteroclinic orbits. SIAM J. Appl. Math., 46, 1057-1078.

Fausch, K. D., Torgersen, C. E., Baxter, C. V., \& Li, H. W. (2002). Landscapes to riverscapes: bridging the gap between research and conservation of stream fishes. Bioscience, 52, 483-498.

Fox, P. J., \& Proctor, M. R. E. (1998). Effects of distant boundaries on pattern forming instabilities. Phys. Rev. E, 57, 491-494.

Fraile, J. M., \& Sabina, J. C. (1989). General conditions for the existence of a critical point-periodic wave front connection for reaction-diffusion systems. Nonlinear Anal.-Theor., 13, 767-786.

Gaylord, B., \& Gaines, S. D. (2000). Temperature or transport? Range limits in marine species mediated solely by flow. Am. Nat., 155, 769-789.

Grimm, V., \& Wissel, C. (1997). Babel, or the ecological stability discussions: an inventory and analysis of terminology and a guide for avoiding confusion. Oecologia, 109, 323-334.

Hauzy, C., Hulot, F. D., \& Gins, A. (2007). Intra and interspecific density-dependent dispersal in an aquatic prey-predator system. J. Anim. Ecol., 76, 552-558.

Hilker, F. M., \& Lewis, M. A. (2010). Predator-prey systems in streams and rivers. Theor. Ecol., 3, 175193.

Huerre, P., \& Monkewitz, P. A. (1990). Local and global instabilities in spatially developing flows. Annu. Rev. Fluid Mech., 22, 473-537.

Huisman, J., \& Weissing, F. J. (1999). Biodiversity of plankton by species oscillations and chaos. Nature, 402, 407-410.

Klausmeier, C. A. (1999). Regular and irregular patterns in semiarid vegetation. Science, 284, 1826-1828.

Kopell, N., \& Howard, L. N. (1973). Plane wave solutions to reaction-diffusion equations. Stud. Appl. Math., 52, 291-328.

Lee, S.-H. (2012). Effects of uniform rotational flow on predator-prey system. Physica A, 391, 6008-6015.

Levine, J. M. (2003). A patch modeling approach to the community-level consequences of directional dispersal. Ecology, 84, 1215-1224.

Lutscher, F., Lewis, M. A., \& McCauley, E. (2006). Effects of heterogeneity on spread and persistence in rivers. Bull. Math. Biol., 68, 2129-2160.

Malchow, H. (2000). Motional instabilities in predator-prey systems. J. Theor. Biol., 204, 639-647.

Malchow, H., \& Petrovskii, S. V. (2002). Dynamical stabilization of an unstable equilibrium in chemical and biological systems. Math. Comput. Model., 36, 307-319.

Merchant, S. M., \& Nagata, W. (2010). Wave train selection behind invasion fronts in reaction-diffusion predator-prey models. Physica D, 239, 1670-1680.

Merchant, S. M., \& Nagata, W. (2011). Instabilities and spatiotemporal patterns behind predator invasions with nonlocal prey competition. Theor. Popul. Biol., 80, 289-297.

Morozov, A. Yu., Petrovskii, S. V, \& Li, B.-L. (2006). Spatiotemporal complexity of the patchy invasion in a predator-prey system with the Allee effect. J. Theor. Biol., 238, 18-35.

Nauman, E. B. (2008). Chemical reactor design, optimization, and scaleup (2nd ed.). Hoboken: Wiley.

Owen, M. R., \& Lewis, M. A. (2001). How predation can slow, stop or reverse a prey invasion. Bull. Math. Biol., 63, 655-684.

Perumpanani, A. J., Sherratt, J. A., \& Maini, P. K. (1995). Phase differences in reaction-diffusionadvection systems and applications to morphogenesis. IMA J. Appl. Math., 55, 19-33.

Petrovskii, S. V., \& Malchow, H. (2000). Critical phenomena in plankton communities: KISS model revisited. Nonlinear Anal., Real World Appl., 1, 37-51.

Petrovskii, S., Li, B.-L., \& Malchow, H. (2004). Transition to spatiotemporal chaos can resolve the paradox of enrichment. Ecol. Complex., 1, 37-47.

Rademacher, J. D. M. (2006). Geometric relations of absolute and essential spectra of wave trains. SIAM J. Appl. Dyn. Syst., 5, 634-649. 
Rademacher, J. D. M., Sandstede, B., \& Scheel, A. (2007). Computing absolute and essential spectra using continuation. Physica D, 229, 166-183.

Rietkerk, M., Boerlijst, M. C., van Langevelde, F., HilleRisLambers, R., van de Koppel, J., Prins, H. H. T., $\&$ de Roos, A. (2002). Self-organisation of vegetation in arid ecosystems. Am. Nat., 160, 524-530.

Rosenzweig, M. L., \& MacArthur, R. H. (1963). Graphical representation and stability conditions of predator-prey interactions. Am. Nat., 97, 209-223.

Rovinsky, A. B., \& Menzinger, M. (1992). Chemical instability induced by a differential flow. Phys. Rev. Lett., 69, 1193-1196.

Sandstede, B. (2002). Stability of travelling waves. In B. Fiedler (Ed.), Handbook of dynamical systems II (pp. 983-1055). Amsterdam: North-Holland.

Sandstede, B., \& Scheel, A. (2000a). Absolute versus convective instability of spiral waves. Phys. Rev. E, 62, 7708-7714.

Sandstede, B., \& Scheel, A. (2000b). Absolute and convective instabilities of waves on unbounded and large bounded domains. Physica D, 145, 233-277.

Scheuring, I., Károlyi, G., Péntek, A., Tel, T., \& Toroczkai, Z. (2000). A model for resolving the plankton paradox: coexistence in open flows. Freshw. Biol., 45, 123-132.

Sherratt, J. A. (2005). An analysis of vegetation stripe formation in semi-arid landscapes. J. Math. Biol., $51,183-197$.

Sherratt, J. A. (2010). Pattern solutions of the Klausmeier model for banded vegetation in semi-arid environments I. Nonlinearity, 23, 2657-2675.

Sherratt, J. A., Lewis, M. A., \& Fowler, A. C. (1995). Ecological chaos in the wake of invasion. Proc. Natl. Acad. Sci. USA, 92, 2524-2528.

Sherratt, J. A., Smith, M. J., \& Rademacher, J. D. M. (2009). Locating the transition from periodic oscillations to spatiotemporal chaos in the wake of invasion. Proc. Natl. Acad. Sci. USA, 106, 10890-10895.

Smith, M. J., \& Sherratt, J. A. (2009). Propagating fronts in the complex Ginzburg-Landau equation generate fixed-width bands of plane waves. Phys. Rev. E, 80, 046209.

Smith, M. J., Sherratt, J. A., \& Lambin, X. (2008). The effects of density-dependent dispersal on the spatiotemporal dynamics of cyclic populations. J. Theor. Biol., 254, 264-274.

Smith, M. J., Rademacher, J. D. M., \& Sherratt, J. A. (2009). Absolute stability of wavetrains can explain spatiotemporal dynamics in reaction-diffusion systems of lambda-omega type. SIAM J. Appl. Dyn. Syst., 8, 1136-1159.

Suslov, S. A. (2001). Searching convective/absolute instability boundary for flows with fully numerical dispersion relation. Comput. Phys. Commun., 142, 322-325.

Suslov, S. A. (2006). Numerical aspects of searching convective/absolute instability transition. J. Comp. Physiol., 212, 188-217.

Suslov, S. A. (2009). Analysis of instability patterns in non-Boussinesq mixed convection using a direct numerical evaluation of disturbance integrals. Comput. Fluids, 38, 590-601.

Suslov, S. A., \& Paolucci, S. (2004). Stability of non-Boussinesq convection via the complex GinzburgLandau model. Fluid Dyn. Res., 35, 159-203.

Tobias, S. M., Proctor, M. R. E., \& Knobloch, E. (1998). Convective and absolute instabilities of fluid flows in finite geometries. Physica D, 113, 43-72.

Turchin, P. (2003). Complex population dynamics. A Theoretical/Empirical synthesis. Princeton: Princeton University Press.

van Saarloos, W. (2003). Front propagation into unstable states. Phys. Rep., 386, 29-222.

Wheeler, P., \& Barkley, D. (2006). Computation of spiral spectra. SIAM J. Appl. Dyn. Syst., 5, 157-177.

Wieters, E. A., Gaines, S. D., Navarrete, S. A., Blanchette, C. A., \& Menge, B. A. (2008). Scales of dispersal and the biogeography of marine predator-prey interactions. Am. Nat., 171, 405-417.

Worledge, D., Knobloch, E., Tobias, S., \& Proctor, M. (1997). Dynamo waves in semi-infinite and finite domains. Proc. R. Soc. Lond. A, 453, 119-143. 